\title{
OP 06 DEVELOPING PATIENT CONTROLLED ANALGESIA (PCA) IN THE HOME FOR CHILDREN AND YOUNG PEOPLE (CYP)
}

T. Boggs ${ }^{1 *}$, D. Rajapske ${ }^{1}$, P. Kelly ${ }^{2}$, D. R. Hollowell ${ }^{2}$, R. Howard ${ }^{3}$, F. Craig ${ }^{1}$, M. BluebondLangner ${ }^{1}$ Louis Dundas Centre for Children's Palliative Care-Haematology/Oncology Outreach and Palliative Care Team, Great Ormond Street Hospital; 2Louis Dundas Centre for Children's Palliative Care, UCL/Institute of Child Health; ${ }^{3}$ Pain Service, Great Ormond Street Hospital; 4 True Colours Chair in Children's Palliative Care, Louis Dundas Centre for Children's Palliative Care, UCL/Institute of Child Health

10.1136/bmjspcare-2012-000264.6

Introduction Pain management for CYP in advanced disease is sub-optimal and, of concern to parents and palliative care professionals. PCA provides the patient, with a mechanism to rapidly and safely self-administer a bolus dose of analgesia in response to their experiences of pain. There is limited evidence on its use for CYP receiving palliative care.

Aims and Methods To identify the salient issues and challenges in developing a home PCA service for CYP patients of a tertiary palliative care (PC) service. A mixed methods processual evaluation including: (a) longitudinal systematic participant observations of multidisciplinary project team planning meetings, PC team rounds and PCA training sessions, (b) interviews with community and tertiary based staff providing palliative care, (c) prospective pain data from CYP and carers (d) retrospective case review of parenteral analgesia utilisation, (e) survey of affiliated community services.

Results We identified the following issues to consider when developing this service: (1) only small numbers of CYP require PCA, limiting the potential for developing and sustaining clinical expertise, (2) introduction of PCA is time sensitive requiring rapid and flexible responses from a range of services, (3) normalisation of PCA as a symptom management technique reduced safety concerns and increased professional, parent and CYP confidence.

Conclusion Preliminary analysis suggests provision of PCA in the home is feasible although resource intensive. Further research is now required to address the efficacy of this symptom management technique, in this patient population. 